\title{
Effect of random weight errors on the performance of partially adaptive array beamformers \#
}

\author{
Shiann-Jeng Yu, Ju-Hong Lee* \\ Department of Electrical Engineering, College of Engineering, National Taiwan University, Taipei, Taiwan, ROC
}

Received 5 January 1993; revised 29 April 1993

\begin{abstract}
This paper analyzes the effect of random weight errors on the performance of array beamformers with partial adaptivity. Based on the structure of the generalized sidelobe canceller (GSC), three different beamformers, namely, element space, eigenspace, and beam space partially adaptive beamformers are considered. Theoretical formulas representing the beamformer's performance sensitivity to random errors of the adaptive weights are derived. These formulas show that the performance sensitivity to the random weight errors increases as the number of the adaptive weights increases in each case of the three different beamformers. Furthermore, we find that the choice of the signal blocking matrix required in the GSC structure also affects the performance of the array beamformers in the presence of random weight errors. Computer simulations confirming the theoretical analyses are provided.
\end{abstract}

\section{Zusammenfassung}

In dieser Arbeit wird die Answirkung zufälliger Gewichtsfehler auf die Wirkungsweise von Arrays mit partieller Adaptivität untersucht. Basierend auf der Struktur der verallgemeinerten Auslöschung von Nebenmaxima werden drei verschiedene Strahlformer betrachtet: Elementraum-, Eigenraum- und Strahlraumformer mit teilweiser Adaptivität. Theoretische Formeln für die Empfindlichkeit bezüglich zufälliger Fehler der adaptiven Gewichte werden abgeleitet. Sie zeigen, daß die Empfindlichkeit bzgl. der zufälligen Gewichtsfehler mit steigender Zahl der adaptiven Gewichte in allen drei Fällen zunimmt. Weiterhin zeigt es sich, daß die benötigte Signalblockmatrix ebenfalls die Wirkungsweise des Strahlformers beeinflußt, falls zufällige Gewichtsfehler vorliegen. Rechnersimulationen, die die theoretischen Untersuchungen bestätigen, werden präsentiert.

\section{Résumé}

Cet article analyse l'effet d'erreurs de pondération aléatoires sur les performances de formateurs de voie en réseau à adaptativité partielle. Sur la bas de la structure de l'annulateur de lobe secondaire généralisé (GSC) trois formateurs de voie différents, à savoir les formateurs partiellement adaptatifs dans l'espace des éléments, dans l'espace propre, et dans l'espace de voie sont considérés. Nous dérivons des formules théoriques représentant la sensibilité des performances du formateur de voie vis-à-vis d'erreurs aléatoires dans les coefficients de pondération adaptatifs. Ces formules montrent que la sensibilité des performances vis-à-vis des erreurs de pondération aléatoires augmente lorsque le nombre de coefficients

\footnotetext{
* Corresponding author.

* This work was supported by the National Science Council under Grant NSC 82-0404-E002-081.
} 
de pondération adaptif augmente dans les trois cas de formateur envisagés. De plus, nous montrons que le choix de la matrice de signal bloquante requise dans la structure GSC affecte également les performances des formateurs vis-à-vis des erreurs de pondération aléatoires. Nous fournissons des résultats de simulation confirmant les analyses théoriques.

Key words: Partially adaptive array beamformers; Generalized sidelobe canceller; Adaptive weights; Random weight errors

\section{Introduction}

Strongly influenced by the recent advances in digital processing technology, the applications of adaptive arrays have been becoming more important and widespread in many areas, such as radar, sonar, and communication systems. Adaptive array beamformer designed for receiving the desired signal in a specified beam direction while rejecting noise and other directional interferences is one of the well-known examples in adaptive array processing. However, two major problems make an adaptive beamformer with full adaptivity prohibitive in these applications. First, the required number of array elements may be as high as in the order of $10^{4}$. This leads to the fact that the required real-time computational complexity may be in the order of $10^{12}$ depending upon the special algorithm used [3]. The other problem concerns the quickly changing interference environments where a fully adaptive beamformer would be unable to adapt fast enough to successfully track the changes. Therefore, array beamformers with partial adaptivity have been receiving much attention. Using partial adaptivity we can reduce the cost and the computational complexity required by a fully adaptive beamformer, since only a part of the available degrees of freedom is adapted. Moreover, the adaptive speed of a partially adaptive beamformer can be increased because the number of the adaptive weights is reduced.

Many partially adaptive beamformers have been proposed in the literature $[1,2,5,8,9,13-16]$. Notable among them is the one which uses the generalized sidelobe canceller (GSC) structure of [6] to achieve partial adaptivity. This partially adaptive beamformer has been considered widely $[5,8,13-16]$ due to its effectiveness and simplicity.

Although adaptive array beamformers have been successfully used for the purpose of beamforming, their performance is very sensitive to the mismatch between the actual weights and the ideal weights. The mismatch may be due to the quantization of weight values. Nitzberg [10] and Godara [4] have analyzed the effect of weight errors on array performance. They show that a fully adaptive array beamformer is very sensitive to random weight errors.

This paper investigates the effect of random errors induced by the adaptive weights on the performance of adaptive array beamformers. Analytical formulas representing the array output SINR (signal-to-interference plus noise power ratio) in the presence of random weight errors are derived for element space, eigenspace, and beam space partially adaptive beamformers, respectively. Similar analytical formulas are also derived for fully adaptive beamformers for comparison. The theoretical results show that adaptive beamformers with partial adaptivity are less sensitive to random weight errors when compared to fully adaptive beamformers with the same array size. We also show that the signal blocking matrix used in the GSC structure of a partially adaptive beamformer affects the array performance when random weight errors exist. Computer simulations confirm the theoretical work.

The paper is organized as follows. Section 2 briefly describes the signal representations and the general formulation for partially adaptive array beamforming. The random errors of the adaptive weights are introduced and the analytical formulas representing their effect on the performance of GSC based adaptive beamformers are derived in Section 3 . From these formulas, the effect of the signal blocking matrix used for the GSC structure is also discussed. Based on the analyses presented in Section 3, we further derive the analytical formulas for representing the effects of random weight errors on element space, eigenspace, and beam space partially adaptive beamformers, respectively, in 
Section 4. Section 5 presents several computer simulations. The theoretical work done in this paper is confirmed by simulation examples. Finally, we conclude the paper in Section 6 .

\section{Formulation of adaptive array beamforming}

Consider an adaptive array beamformer based on the GSC structure with $N$ elements as shown in Fig. 1. This structure of adaptive array beamformers was proposed and shown effective in [6]. Let the received signal data vector be $X(t)=$ $S(t)+S_{\mathrm{i}}(t)+N(t)$, where $S(t)$ is the desired signal vector with power $p_{\mathrm{s}}, S_{\mathrm{i}}(t)$ is the interference signal vector with correlation matrix $R_{\mathfrak{i}}$, and $N(t)$ is the white noise vector with correlation matrix $\sigma^{2} I$. The output signal $d(t)$ of the upper branch is given by

$d(t)=W_{\mathrm{q}}^{\mathrm{H}} X(t)$.

The quiescent weight vector $W_{\mathrm{q}}$ is chosen such that the output signal power $E\left[|d(t)|^{2}\right]$ is minimized subject to a set of $L$ linear constraints. That is,

Minimize $E\left[|d(t)|^{2}\right]$ subject to $C^{\mathrm{H}} W_{\mathrm{q}}=f$,

where $C$ denotes the constraint matrix with size $N \times L$ and $f$ is an $L \times 1$ response vector, the superscript $\mathrm{H}$ denotes Hermitian operation. Suppose that the received data are composed of white noise only. Then $W_{\mathrm{q}}$ is given by

$W_{\mathrm{q}}=C\left(C^{\mathbf{H}} C\right)^{-1} f$.
The overall array weight vector is given by $W=W_{\mathrm{q}}-B W_{\mathrm{a}}$, where $B$ is the $N \times(N-L)$ signal blocking matrix which blocks out the desired signal in the signal vector $U(t)$ of the lower branch. Conventionally, $B$ is designed to have the properties that $B^{\mathrm{H}} C=0$ and rank of $B=N-L$. The optimal adaptive weight vector $W_{\mathrm{a}}$ of the lower branch is given by

$$
W_{\mathrm{a}}=R_{U}^{-1} P \text {, }
$$

which is found by minimizing the overall array output power given by

$$
\begin{aligned}
\zeta_{\mathrm{f}}=E\left[|y(t)|^{2}\right] & =E\left[\left|W_{\mathbf{q}}^{\mathrm{H}} X(t)-W_{\mathrm{af}}^{\mathrm{H}} U(t)\right|^{2}\right] \\
& =E\left[\left|W_{\mathbf{q}}^{\mathrm{H}} X(t)\right|^{2}\right]-P^{\mathrm{H}} R_{U}^{-1} P,
\end{aligned}
$$

where $R_{U}=B^{\mathrm{H}} R_{X} B=B^{\mathrm{H}} R_{\mathrm{i}} B+\sigma^{2} B^{\mathrm{H}} B$ is the correlation matrix of the output signal vector $U(t)=B^{\mathbf{H}} X(t)$ of the signal blocking matrix $B$, $P=B^{\mathrm{H}} R_{X} W_{\mathrm{q}}$, and $R_{X}=E\left\{X(t) X^{\mathrm{H}}(t)\right\}$ is the correlation matrix of the received signal vector $X(t)$. The subscript 'af' in (5) designates the adaptive weights obtained without considering partial adaptivity, i.e., $W_{\text {af }}$ is the adaptive weight vector associated with the GSC based array beamformers with full adaptivity.

Next, consider that a partially adaptive beamformer uses a part of $U(t)$ to find the optimal adaptive weights. Suppose that $M(M<N-L)$ signals of the signal vector $U(t)$ are used. Let the matrix $F$ with size $(N-L) \times M$ span the signal subspace associated with the $M$ chosen signals and $G$ with size $(N-L) \times(N-L-M)$ span

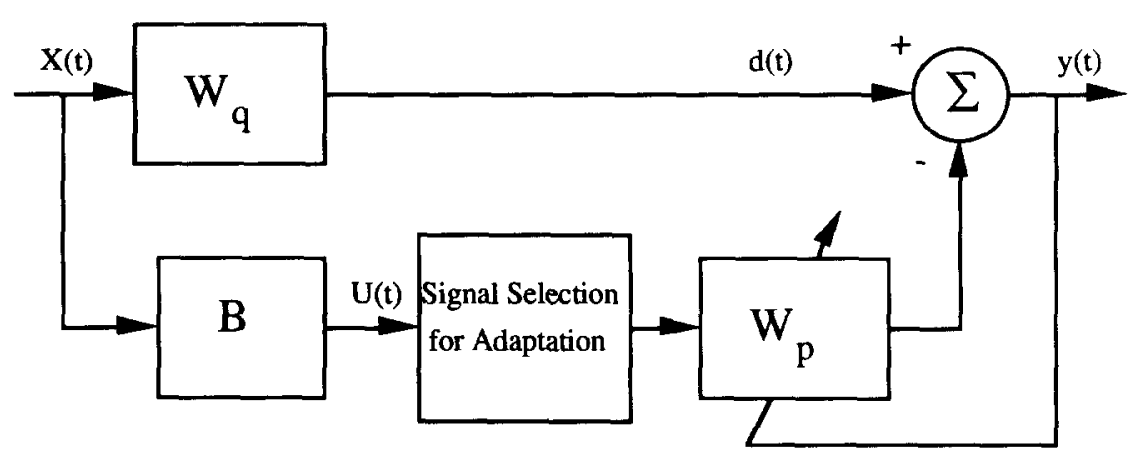

Fig. 1. The configuration of the adaptive array beamformers based on GSC structure. 
the unused signal subspace. Hence, rank of $[F \mid G]=(N-L)$. Then the optimal weight vector $W_{\mathrm{a}}$ is obtained by solving the following minimization problem:

Minimize $E\left[|y(t)|^{2}\right] \quad$ subject to $G^{\mathbf{H}} W_{\mathbf{a}}=0$.

Its solution is given by

$W_{\mathrm{ap}}=W_{\mathrm{af}}-R_{U}^{-1} G\left(G^{\mathrm{H}} R_{U}^{-1} G\right)^{-1} G^{\mathrm{H}} W_{\mathrm{af}}$.

Substituting (7) into $y(t)=d(t)-W_{\mathrm{ap}}^{\mathrm{H}} U(t)$ and computing the power of $y(t)$ yields

$\zeta_{\mathrm{p}}=\zeta_{\mathrm{f}}+P^{\mathrm{H}} R_{U}^{-1} G\left(G^{\mathrm{H}} R_{U}^{-1} G\right)^{-1} G^{\mathrm{H}} R_{U}^{-1} P$.

Comparing (5) and (8), we note that $\zeta_{\mathrm{p}}>\zeta_{\mathrm{f}}$. That is, the GSC based array beamformers with partial adaptivity produce larger output power than that of the GSC based array beamformers with full adaptivity.

\section{Effect of random weight errors}

Here, we assume that the computed adaptive weights are different from the optimal adaptive weights shown as follows:

$\hat{W}_{\mathrm{a}}=W_{\mathrm{a}}+\Gamma_{\mathrm{w}}$,

where $\Gamma_{\mathrm{w}}$ denotes the weight error vector. Its elements $\gamma_{i}, i=1,2, \ldots, N-L$, are statistically independent complex random variables with mean zero and variance $\sigma_{\mathrm{w}}^{2}$. The overall array weight vector then becomes $W=W_{\mathrm{q}}-B W_{\mathrm{a}}=W-B \Gamma_{\mathrm{w}}$, where $W=W_{\mathrm{q}}-B W_{\mathrm{a}}$. Since $S(t), S_{\mathrm{i}}(t)$, and $N(t)$ are mutually uncorrelated, the array output power is given by

$\hat{\zeta}=\hat{W}^{\mathbf{H}} R_{X} \hat{W}=\hat{\zeta}_{\mathrm{s}}+\hat{\zeta}_{\mathrm{i}}+\hat{\zeta}_{\mathrm{n}}$,

where $\hat{\zeta}_{\mathrm{s}}=\hat{W}^{\mathbf{H}} E\left\{S(t) S(t)^{\mathbf{H}}\right\} \hat{W}$ and its expectation is the output signal power which is the same as that of the GSC based array beamformers without random errors in $W_{a}, \hat{\zeta}_{i}$ is the output interference power given by

$$
\begin{aligned}
\hat{\zeta_{\mathrm{i}}}= & \hat{W}^{\mathrm{H}} E\left\{S_{\mathrm{i}}(t) S_{\mathrm{i}}(t)^{\mathrm{H}}\right\} \hat{W} \\
= & \left(W-B \Gamma_{\mathrm{w}}\right)^{\mathrm{H}} R_{\mathrm{i}}\left(W-B \Gamma_{\mathrm{w}}\right) \\
= & W^{\mathrm{H}} R_{\mathrm{i}} W+\Gamma_{\mathrm{w}}^{\mathrm{H}} B^{\mathrm{H}} R_{\mathrm{i}} B \Gamma_{\mathrm{w}} \\
& -\Gamma_{\mathrm{w}}^{\mathrm{H}} B^{\mathrm{H}} R_{\mathrm{i}} W-W^{\mathrm{H}} R_{\mathrm{i}} B \Gamma_{\mathrm{w}} .
\end{aligned}
$$

$\hat{\zeta}_{\mathrm{n}}$ is the output noise power given by

$$
\hat{\zeta}_{\mathrm{n}}=\sigma^{2}\left(W^{\mathrm{H}} W+\Gamma_{\mathrm{w}}^{\mathrm{H}} B^{\mathrm{H}} B \Gamma_{\mathrm{w}}\right.
$$$$
\left.-W^{\mathrm{H}} B \Gamma_{\mathrm{w}}-\Gamma_{\mathrm{w}}^{\mathrm{H}} B^{\mathrm{H}} W\right) \text {. }
$$

Taking the expectations of $\hat{\zeta}_{\mathrm{i}}$ and $\hat{\zeta}_{\mathrm{n}}$, we obtain

$E\left\{\hat{\zeta}_{\mathrm{i}}\right\}=\hat{\zeta}_{\mathrm{i}}^{\prime}=W^{\mathrm{H}} R_{\mathrm{i}} W+E\left\{\Gamma_{\mathrm{w}}^{\mathrm{H}} B^{\mathrm{H}} R_{\mathrm{i}} B \Gamma_{\mathrm{w}}\right\}$

and

$E\left\{\hat{\zeta}_{\mathrm{n}}\right\}=\hat{\zeta}_{\mathrm{n}}^{\prime}=\sigma^{2}\left(W^{\mathrm{H}} W+E\left\{\Gamma_{\mathrm{w}}^{\mathrm{H}} B^{\mathrm{H}} B \Gamma_{\mathrm{w}}\right\}\right)$,

respectively. Since the term $W^{\mathrm{H}} R_{\mathrm{i}} W$ represents the output interference power without the random weight errors, it is negligible. The output SINR is then approximately given by

$$
\begin{aligned}
& \operatorname{SINR}=\frac{p_{\mathrm{s}}}{\sigma^{2} W^{\mathrm{H}} W} \\
& \times\left[1+\frac{\operatorname{Tr}\left(B^{\mathrm{H}} B E\left\{\Gamma_{\mathrm{w}} \Gamma_{\mathrm{w}}^{\mathrm{H}}\right\}\right)+\sigma^{-2} \operatorname{Tr}\left(B^{\mathrm{H}} R_{\mathrm{i}} B E\left\{\Gamma_{\mathrm{w}} \Gamma_{\mathrm{w}}^{\mathrm{H}}\right\}\right)}{W^{\mathrm{H}} W}\right]^{-1} \\
&=\frac{p_{\mathrm{s}}}{\sigma^{2} W^{\mathrm{H} W}}\left[1+\frac{\sigma^{-2} \operatorname{Tr}\left(R_{U} E\left\{\Gamma_{\mathrm{w}} \Gamma_{\mathrm{w}}^{\mathrm{H}}\right\}\right)}{W^{\mathrm{H}} W}\right]^{-1},
\end{aligned}
$$

where $p_{\mathrm{s}} / \sigma^{2} W^{\mathrm{H}} W$ is the output SINR of the adaptive array beamformer without random weight errors. ' $T r$ ' denotes trace operation. In the case of GSC based fully adaptive beamformers, i.e., $M=N-1, \Gamma_{\mathrm{w}}$ is the weight error vector with covariance matrix given by

$E\left\{\Gamma_{\mathrm{w}} \Gamma_{\mathrm{w}}^{\mathrm{H}}\right\}=\sigma_{\mathrm{w}}^{2} I$.

Then (15) becomes

$\operatorname{SINR}=\frac{p_{\mathrm{s}}}{\sigma^{2} W^{\mathrm{H}} W}\left[1+\sigma_{\mathrm{w}}^{2} \frac{\sigma^{-2} \operatorname{Tr}\left(R_{U}\right)}{W^{\mathrm{H}} W}\right]^{-1}$.

For the purpose of comparing array sensitivity to random weight errors, we define a weight degrading factor (WDF) as the inverse term in (15). That is,

$\mathrm{WDF}=\left[1+\frac{\sigma^{-2} \operatorname{Tr}\left(R_{U} E\left\{\Gamma_{\mathrm{w}} \Gamma_{\mathrm{w}}^{\mathbf{H}}\right\}\right)}{W^{\mathrm{H}} W}\right]^{-1}$.

Note that the value of the WDF shown in (18) is always less than one. As a result, the output SINR of an adaptive array beamformer with random weight errors given in (15) is always less than that of the adaptive array beamformer without random 
weight errors. Moreover, (18) reveals that the signal blocking matrix $B$ affects the array performance. Hence, how to design an appropriate signal blocking matrix may have to be considered when random weight errors exist. To reduce the effect of $B$ on the value of WDF, we may change $B$ to $\delta B$ with $0<\delta<1$. If random weight errors do not exist, it can be easily shown that reducing the value of $\delta$ does not affect the optimal weight vector $W$ but increases the magnitude of the adaptive weight vector $W_{\mathrm{a}}$. This leads to alleviating the effect of random weight errors due to quantization errors.

Next, we evaluate the sensitivity of adaptive array beamformers to the random weight errors. The $W^{\mathrm{H}} W$ is given by

$W_{\mathrm{f}}^{\mathrm{H}} W_{\mathrm{f}}=f^{\mathrm{H}}\left(C^{\mathrm{H}} C\right)^{-1} f+W_{\mathrm{af}}^{\mathrm{H}} B^{\mathrm{H}} B W_{\mathrm{af}}$

for beamformers with full adaptivity, while it is approximately given by

$$
\begin{aligned}
W_{\mathrm{p}}^{\mathrm{H}} W_{\mathrm{p}}= & f^{\mathrm{H}}\left(C^{\mathrm{H}} C\right)^{-1} f+W_{\mathrm{af}}^{\mathrm{H}} B^{\mathrm{H}} B W_{\mathrm{af}} \\
& +\sigma^{-2} W_{\mathrm{af}}^{\mathrm{H}} G\left(G^{\mathrm{H}} R_{U}^{-1} G\right)^{-1} G^{\mathrm{H}} W_{\mathrm{af}} \\
= & W_{\mathrm{f}}^{\mathrm{H}} W_{\mathrm{f}}+\sigma^{-2} W_{\mathrm{af}}^{\mathrm{H}} G\left(G^{\mathrm{H}} R_{U}^{-1} G\right)^{-1} G^{\mathrm{H}} W_{\mathrm{af}}
\end{aligned}
$$

for partially adaptive beamformers if the interference signals are almost cancelled. Obviously, the value of (20) is greater than that of (19). Moreover, the weight error vector $\Gamma_{\mathrm{w}}$ has covariance matrix given by

$E\left\{\Gamma_{\mathrm{w}} \Gamma_{\mathrm{w}}^{\mathrm{H}}\right\}=\sigma_{\mathrm{w}}^{2} \Lambda_{\mathrm{w}}$,

where $\Lambda_{w}$ is a diagonal matrix with 1 located at the positions corresponding to the $M$ chosen signal channels and zero elsewhere for partially adaptive beamformers. Therefore, we have

$\operatorname{Tr}\left[B^{\mathrm{H}} B A_{\mathrm{w}}\right] \leqslant \operatorname{Tr}\left[B^{\mathrm{H}} B\right]$

and

$\operatorname{Tr}\left[B^{\mathrm{H}} R_{\mathrm{i}} B \Lambda_{\mathrm{w}}\right] \leqslant \operatorname{Tr}\left[B^{\mathrm{H}} R_{\mathrm{i}} B\right]$.

Substituting Eqs. (19)-(23) into (18) for fully and partially adaptive beamformers, respectively, we obtain the following relationship:

$\mathrm{WDF}_{\mathrm{f}} \leqslant \mathrm{WDF}_{\mathrm{p}} \leqslant 1$,

where the subscripts $f$ and $p$ denote the cases of fully and partially adaptive beamformers, respectively. Eq. (24) shows that the WDF of a partially adaptive beamformer is greater than that of a fully adaptive beamformer. In other words, a partially adaptive beamformer is less sensitive to random weight errors compared to a fully adaptive beamformer with the same array size.

\section{Sensitivity of partially adaptive beamformers}

In this section, we derive the weight degrading factor (WDF) of several GSC based partially adaptive beamformers, namely, element space, eigenspace, and beam space partially adaptive beamformers which have been widely considered in the literature. Their sensitivities to random weight errors are also compared with those of fully adaptive beamformers in terms of the WDF.

\subsection{Element space partially adaptive beamformers}

A systematic method in constructing $B$ for equally-spaced array beamformers with linear or derivative constraints was proposed in [7]. Suppose that there are $L$ linear or derivative constraints used for adaptive beamforming. The associated signal blocking matrix $B$ can be constructed as follows:

$\boldsymbol{B}^{\mathbf{H}}=\left[\begin{array}{ccc}b^{\mathbf{H}} & & 0 \\ & \ddots & \\ 0 & & b^{\mathbf{H}}\end{array}\right]$,

where $B$ is a Toeplitz matrix with vector entry $b^{\mathbf{H}}=\left[b_{0}, b_{1}, \ldots, b_{\mathbf{L}}\right]$. In the case of unit gain constraint, $L=1$ and the vector entry is given by

$b^{\mathbf{H}}=\left[\begin{array}{ll}1 & -1\end{array}\right]$.

The corresponding signal blocking matrix $B$ is of the form

$\boldsymbol{B}^{\mathbf{H}}=\left[\begin{array}{rrrrrrr}1 & -1 & 0 & 0 & \cdots & 0 & 0 \\ 0 & 1 & -1 & 0 & \cdots & 0 & 0 \\ . & . & . & . & & . & . \\ 0 & 0 & 0 & 0 & \cdots & 1 & -1\end{array}\right]$. 
Assume that there are $J$ interferers. The interference covariance matrix $R_{\mathrm{i}}$ can be written as

$R_{\mathrm{i}}=\sum_{i=1}^{J} p_{i} S_{i} S_{i}^{\mathrm{H}}$

where $p_{i}$ and $S_{i}$ are the power and phase vector of the $i$ th interferer, respectively. From (27) and (28), we obtain

$B^{\mathrm{H}} R_{\mathrm{i}} B=\sum_{i=1}^{J} \alpha_{i} p_{i} S_{i}^{\prime} S_{i}^{\prime \mathrm{H}}$

where $\alpha_{i}=\left|1-\mathrm{e}^{\mathrm{j} \phi_{i}}\right|^{2}$ and $\phi_{i}$ is the phase associated with the $i$ th interferer. $S_{i}^{\prime}$ denotes the phase vector associated with the $i$ th interferer with size less than that of $S_{i}$ by one. Furthermore, we have

$\operatorname{Tr}\left(B^{\mathrm{H}} B E\left\{\Gamma_{\mathrm{w}} \Gamma_{\mathrm{w}}^{\mathrm{H}}\right\}\right)=2 M \sigma_{\mathrm{w}}^{2}$,

where $M$ is the number of signals selected from the signal vector $U(t)$ and it is called the degrees of freedom for weight adaptation. From (29), we have

$\operatorname{Tr}\left(B^{\mathrm{H}} R_{\mathrm{i}} B E\left\{\Gamma_{\mathrm{w}} \Gamma_{\mathrm{w}}^{\mathbf{H}}\right\}\right)=M \sigma_{\mathrm{w}}^{2} \sum_{i=1}^{J} p_{i} \alpha_{i}$

Substituting (30) and (31) into the WDF of (18) yields

$\mathrm{WDF}=\left[1+M \sigma_{\mathrm{w}}^{2}\left(\frac{2+\sum_{i=1}^{J} \mathrm{INR}_{i} \alpha_{i}}{W^{\mathrm{H}} W}\right)\right]^{-1}$,

where $I_{N R}$ is the $i$ th interference to noise power ratio. (32) shows that the WDF may be dependent of the signal channels chosen from $U(t)$ for weight adaptation. For the GSC based beamformers with full adaptivity, $M$ is equal to $N-1$ and the $W^{\mathrm{H}} W$ is given by (19). In contrast, $M<N-1$ and the $W^{\mathrm{H}} W$ is given by (20) for the GSC based beamformers with partial adaptivity. Therefore, we can see that $\mathrm{WDF}_{\mathrm{f}} \leqslant \mathrm{WDF}_{\mathrm{p}} \leqslant 1$.

If the first derivative constraint is imposed in addition to the unit gain constraint, then $b^{\mathrm{H}}$ is given by

$b^{\mathbf{H}}=\left[\begin{array}{lll}1 & -2 & 1\end{array}\right]$ and the signal blocking matrix $B$ is given by

$$
B^{\mathrm{H}}=\left[\begin{array}{rrrrrrrrr}
1 & -2 & 1 & 0 & 0 & \cdots & 0 & 0 & 0 \\
0 & 1 & -2 & 1 & 0 & \cdots & 0 & 0 & 0 \\
& \ldots & & & \ldots & & & \ldots & \\
0 & 0 & 0 & 0 & 0 & \cdots & 1 & -2 & 1
\end{array}\right] .
$$

Following the same procedure as for the case of unit gain constraint, we can easily obtain the corresponding WDF as follows:

$\mathrm{WDF}=\left[1+M \sigma_{\mathrm{w}}^{2}\left(\frac{6+\sum_{i=1}^{J} \mathrm{INR}_{i} \alpha_{i}^{2}}{W^{\mathrm{H}} W}\right)\right]^{-1}$.

For the GSC based beamformers with full adaptivity, $M=N-2$ and $W^{\mathrm{H}} W$ is given by (19). $M<N-2$ and the $W^{\mathrm{H}} W$ is given by (20) for the GSC based beamformers with partial adaptivity. Again, we note that $\mathrm{WDF}_{\mathrm{f}} \leqslant \mathrm{WDF}_{\mathrm{p}} \leqslant 1$.

Comparing (32) and (35), we note that using different signal blocking matrices may result in different array's sensitivities to random weight errors. Moreover, the WDF may decrease as the number of constraints used increases. This indicates that the derivative constraints widely used for improving the array gain in the presence of steering vector errors may degrade the array performance when random weight errors exist.

\subsection{Eigenspace partially adaptive beamformers}

Array beamformers adopting eigenspace criterion for the selection of partial adaptivity are discussed in [14] in detail. In this case, the signal blocking matrix $B$ is chosen to satisfy

$B^{\mathrm{H}} B=I \quad$ and $\quad B^{\mathrm{H}} C=0$.

Therefore, we choose the eigenvectors of $C\left(C^{\mathrm{H}} C\right)^{-1} C^{\mathrm{H}}$ corresponding to zero eigenvalues as the columns of $B$ in our computer simulations for this case.

Next, let the eigendecomposition of $R_{U}$ be expressed as

$$
R_{U}=\sum_{i=1}^{N-L} \lambda_{i} e_{i} e_{i}^{\mathrm{H}}=E D E^{\mathrm{H}},
$$


where matrix $D=\operatorname{diag}\left\{\lambda_{1}, \lambda_{2}, \ldots, \lambda_{N-L}\right\}$ with $\lambda_{1} \geqslant \lambda_{2} \geqslant \cdots \geqslant \lambda_{N-L}$ and $\lambda_{i}$ is the $i$ th eigenvalue of $R_{U}$ corresponding to the $i$ th eigenvector $e_{i}$. Matrix $E=\left[e_{1}, e_{2}, \ldots, e_{N-L}\right]$. To design a partially adaptive beamformer with $M(M<N-L)$ degrees of freedom, [14] has proposed to choose the $M$ eigenvectors such that the normalized sum of their eigenvalues satisfies

$$
\sum_{i=1}^{M} \lambda_{i} / \sum_{i=1}^{N-L} \lambda_{i}=\kappa \geqslant \kappa_{1}
$$

where $\kappa_{1}$ is a preset threshold. Combining the signal blocking matrix $B$ and the matrix $E$ produces the overall blocking matrix $T=B E$ for eigenspace partially adaptive beamformers. With the $B$ replaced by the $T$, (7) becomes

$$
W_{\text {ap }}=D^{-1} P-D^{-1} G\left(G^{\mathrm{H}} D^{-1} G\right)^{-1} G^{\mathrm{H}} D^{-1} P
$$

and the corresponding array output power shown in (8) becomes

$\zeta_{\mathrm{p}}=\zeta_{\mathrm{f}}+P^{\mathrm{H}} D^{-1} G\left(G^{\mathrm{H}} D^{-1} G\right)^{-1} G^{\mathrm{H}} D^{-1} P$,

where $P=E^{\mathrm{H}} B^{\mathrm{H}} R_{X} W_{\mathrm{q}}$.

Next, we evaluate the WDF in this case. From (37), we note that $\operatorname{Tr}\left(R_{U}\right)=\operatorname{Tr}\left(E D E^{\mathrm{H}}\right)=\operatorname{Tr}(D)$. The WDF for fully adaptive beamformers then becomes

$\mathrm{WDF}_{\mathrm{f}}=\left[1+\sigma_{\mathrm{w}}^{2}\left(\frac{\sigma^{-2} \operatorname{Tr}(D)}{W_{\mathrm{f}}^{\mathrm{H}} W_{\mathrm{f}}}\right)\right]^{-1}$

with $W_{\mathrm{f}}=W_{\mathrm{q}}-T D^{-1} P$ and $W_{\mathrm{f}}^{\mathrm{H}} W_{\mathrm{f}}$ is equal to that of (19). Since $\operatorname{Tr}\left(D \Lambda_{\mathrm{w}}\right)=\kappa \operatorname{Tr}(D)$, the WDF for partially adaptive beamformers becomes

$\mathrm{WDF}_{\mathrm{p}}=\left[1+\sigma_{\mathrm{w}}^{2}\left(\frac{\sigma^{-2} \kappa \operatorname{Tr}(D)}{W_{\mathrm{p}}^{\mathrm{H}} W_{\mathrm{p}}}\right)\right]^{-1}$

with $W_{\mathrm{p}}=W_{\mathrm{q}}-T W_{\mathrm{ap}}$ and $W_{\mathrm{p}}^{\mathrm{H}} W_{\mathrm{p}}$ is equal to that of (20), where $W_{\text {ap }}$ is given by (39). In the case of GSC based eigenspace adaptive beamforming without random weight errors, it has been shown in [14] that a partially adaptive beamformer has almost the same performance as that of a fully adaptive beamformer with the same array size when $M$ is not less than $J$. This reveals that the values of $W_{\mathrm{f}}^{\mathrm{H}} W_{\mathrm{f}}$ and $W_{\mathrm{p}}^{\mathrm{H}} W_{\mathrm{p}}$ are almost the same. Since the ratio $\kappa$ shown in (38) is not greater than 1 and $M<N-L$, we thus have from (41) and (42) that

$\mathrm{WDF}_{\mathrm{f}} \leqslant \mathrm{WDF}_{\mathrm{p}} \leqslant 1$.

Eq. (43) shows that an eigenspace partially adaptive beamformer is less sensitive to random weight errors than an eigenspace fully adaptive beamformer.

\subsection{Beam space partially adaptive beamformers}

The basic concept of beam space partially adaptive beamformers was introduced in [12]. The received signal vector is passed through a beam selector for choosing the signal channels attached with adaptive weights. The criterion for choosing the signal channels is based on the received power of each signal channel. The signal channels whose powers are higher than a prescribed level shall be selected. The threshold is generally defined in terms of the power of the desired signal to be received. [12] proposed to start choosing the signal channel with the largest signal beam power. The number of chosen signal channels depends on the satisfactoriness of the beamformer's performance. The structure of a beam space partially adaptive beamformer is shown in Fig. 2. The beam selector is a set of linear combiners between the array elements and the array processor. The beam selector operates the received signal vector $X(t)$ to produce a new signal vector $\hat{X}(t)$ according to

$\hat{X}(t)=\left[\hat{x}_{1}(t), \hat{x}_{2}(t), \ldots, \hat{x}_{N}(t)\right]^{\mathrm{T}}=V^{\mathrm{T}} X(t)$,

where the matrix $V$ is given by [3]

$V=\left[V_{1}, V_{2}, \ldots, V_{N}\right]$.

Let the array elements be isotropic and half a wavelength apart. The element vectors $V_{i}$ can be chosen as

$V_{i}=\left[1, \mathrm{e}^{\mathrm{j} \phi_{i}}, \mathrm{e}^{\mathrm{j} 2 \phi_{i}}, \ldots, \mathrm{e}^{\mathrm{j}(N-1) \phi_{i}}\right]^{\mathrm{T}}$

for $i=1,2, \ldots, N$. These $V_{i}$ are linearly independent if $\phi_{i}$ are different (modulo $2 \pi$ ) for different $i$. Hence, the $V_{i}$ form a series of narrowbeam patterns with each in a different direction without affecting the output SINR of the array beamformer. Moreover, the pattern for each $\hat{x}_{i}(t)=V_{i}^{\mathrm{T}} X(t)$ has a beam 


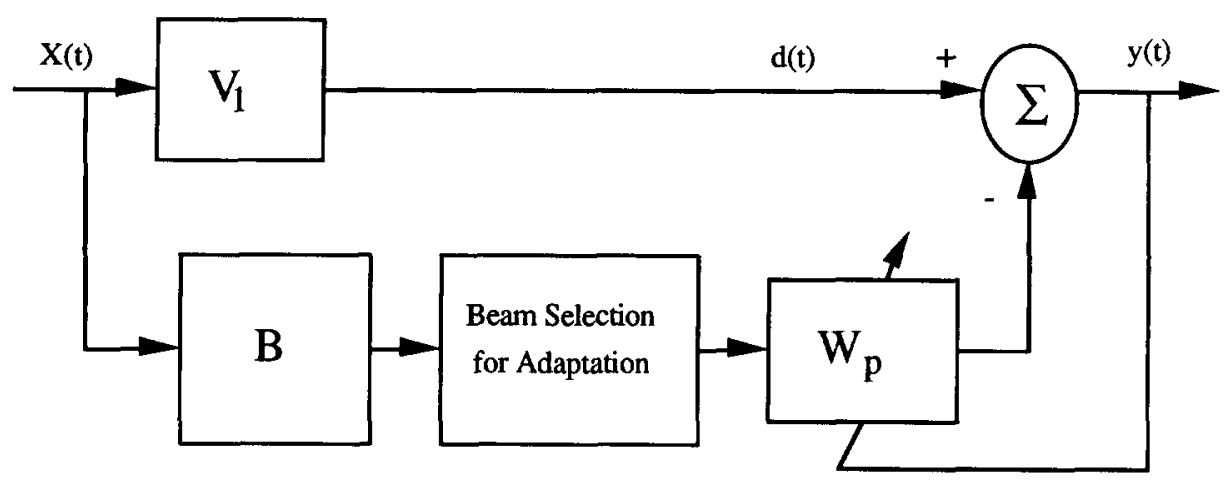

Fig. 2. The GSC based beam space partially adaptive beamformers.

maximum in a direction $\theta_{i}$ which is related to $\phi_{i}$ by

$\phi_{i}=\pi \sin \theta_{i}$.

Next, suppose that the desired signal propagating into the array beamformer from a direction angle off broadside produces a phase vector $S_{\mathrm{s}}$. Let the $V_{1}=S_{\mathrm{s}}$. For the case of unit gain constraint, the $V_{1}$ is set to $S_{\mathrm{s}} / N$. Then the signal blocking matrix $B$ can be set to $B=\left[V_{2}, \ldots, V_{N}\right]$ since these $\phi_{i}$ must be chosen so that

$$
\sum_{k=1}^{N} \exp \left(-\mathrm{j}(k-1) \phi_{i}\right)=0
$$

for all $i \neq 1$ [3]. Substituting this signal blocking matrix $B$ into (18) gives the corresponding WDF as

$\mathrm{WDF}_{\mathrm{f}}=\left[1+\sigma_{\mathrm{w}}^{2}\left(\frac{\sigma^{-2} \operatorname{Tr}\left(R_{U}\right)}{W_{\mathrm{f}}^{\mathrm{H}} W_{\mathrm{f}}}\right)\right]^{-1}$

for beam space beamformers with full adaptivity ( $M=N-1)$, where $W_{\mathrm{f}}^{\mathrm{H}} W_{\mathrm{f}}$ is given by (19), and

$$
\begin{aligned}
\mathrm{WDF}_{\mathrm{p}} & =\left[1+\sigma_{\mathrm{w}}^{2}\left(\frac{\sigma^{-2} \operatorname{Tr}\left(R_{U} \Lambda_{\mathrm{w}}\right)}{W_{\mathrm{p}}^{\mathrm{H}} W_{\mathrm{p}}}\right)\right]^{-1} \\
& =\left[1+\sigma_{\mathrm{w}}^{2}\left(\frac{\sigma^{-2} \beta \operatorname{Tr}\left(R_{U}\right)}{W_{\mathrm{p}}^{\mathrm{H}} W_{\mathrm{p}}}\right)\right]^{-1}
\end{aligned}
$$

for beam space beamformers with partial adaptivity $(M<N-1)$, where $W_{\mathrm{p}}^{\mathrm{H}} W_{\mathrm{p}}$ is given by (20) and
$\operatorname{Tr}\left[R_{U} \Lambda_{\mathrm{w}}\right] / \operatorname{Tr}\left[R_{U}\right]=\beta$. Again, we observe that $\mathrm{WDF}_{\mathrm{f}} \leqslant \mathrm{WDF}_{\mathrm{p}} \leqslant 1$ since we have shown before that $W_{\mathrm{p}}^{\mathrm{H}} W_{\mathrm{p}} \geqslant W_{\mathrm{f}}^{\mathrm{H}} W_{\mathrm{f}}$ and $\beta \leqslant 1$.

\section{Computer simulations}

In this section, several simulation examples demonstrating the effect of random weight errors for partially adaptive beamformers are presented. For all simulations, we consider an equally spaced linear array with 10 elements and half a wavelength for interelement spacing. A desired signal is at broadside with $\mathrm{SNR}=0 \mathrm{~dB}$. For the examples with the first derivative constraint, the phase reference point is located at the array center. In the simulations of random weight errors due to quantization errors, $K$ bits including $K-1$ magnitude bits and one sign bit are used to represent the real and imaginary parts of each adaptive weight. Let the step size of the quantization be $q$. Then the maximum value for representing the real and imaginary parts is given by $q 2^{K-1}=\eta$. The variance associated with the resulting weight error is given by $\sigma_{\mathrm{w}}^{2}=q^{2} / 6$ under the assumption that the quantization errors are independent and uniformly distributed [10].

For each quantization example, the value of the theoretical WDF is computed from the corresponding formula derived in Section 4 using the ideal signal covariance matrix $R_{X}$ and the given $\sigma_{w}^{2}$. The theoretical output SINR (signal-to-interference 

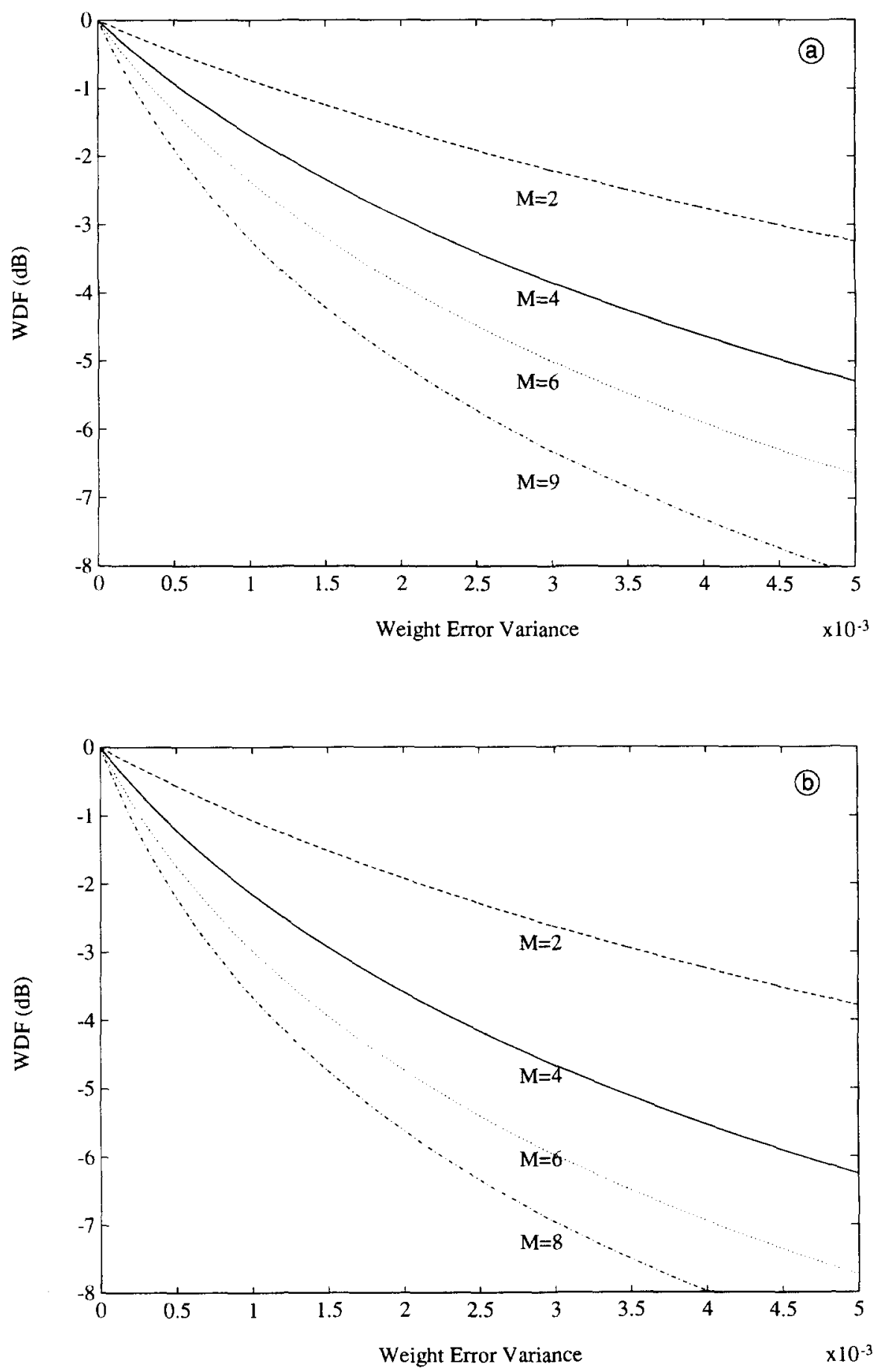

Fig. 3. The WDF versus the weight error variance $\sigma_{w}^{2}$ for the element space beamformer. (a) Unit gain constraint. (b) Unit gain plus 1st derivative constraint. 
Table 1

Effect of quantization errors for the case of element space partially adaptive beamformers

\begin{tabular}{|c|c|c|c|c|}
\hline \multirow[b]{2}{*}{$\begin{array}{l}\text { Number of } \\
\text { bits } K\end{array}$} & \multicolumn{2}{|c|}{ Full adaptivity $(M=9)$} & \multicolumn{2}{|c|}{ Partial adaptivity $(M=6)$} \\
\hline & $\begin{array}{l}\text { Simulation result } \\
\text { SINR }=9.55 \mathrm{~dB} \\
\text { WDF }(\mathrm{dB})\end{array}$ & $\begin{array}{l}\text { Theoretical result } \\
\text { SINR }=9.90 \mathrm{~dB} \\
\text { WDF }(\mathrm{dB})\end{array}$ & $\begin{array}{l}\text { Simulation result } \\
\text { SINR }=9.63 \mathrm{~dB} \\
\text { WDF }(\mathrm{dB})\end{array}$ & $\begin{array}{l}\text { Theoretical result } \\
\text { SINR }=9.85 \mathrm{~dB} \\
\text { WDF }(\mathrm{dB})\end{array}$ \\
\hline 11 & 0 & 0 & 0 & 0 \\
\hline 9 & -0.0003 & -0.0005 & -0.0001 & -0.0003 \\
\hline 7 & -0.0077 & -0.0077 & -0.0061 & -0.0051 \\
\hline 5 & -0.1172 & -0.1223 & -0.0789 & -0.0811 \\
\hline 3 & -1.3631 & -1.6344 & -0.9890 & -1.1448 \\
\hline
\end{tabular}

plus noise ratio) is computed based on the use of the ideal signal covariance matrix $R_{X}$. In contrast, by assuming that ergodicity exists in signal characteristics, 100 independent runs were performed using the sample signal covariance matrix computed from 1000 snapshots to obtain the simulation results. The value of the simulation WDF is the average degradation due to the direct truncation of adaptive weights and the simulation output SINR is the average of all the simulation output SINRs.

\subsection{Examples of element space partially adaptive beamformers}

In this case, an incoherent interferer is incident from $20^{\circ}$ off broadside with INR $=10 \mathrm{~dB}$. Fig. 3(a) shows the WDF in $\mathrm{dB}$ versus the error variance $\sigma_{\mathrm{w}}^{2}$ for the case of the beamformer with unit gain constraint, while Fig. 3(b) shows the simulation results for the case of the beamformer with unit gain plus 1st derivative constraint. Ideal signal covariance matrix $R_{X}$ was used for the above simulations. The curves of $M=9$ and $M=8$ correspond to the GSC based fully adaptive beamformers with unit gain and unit gain plus 1st derivative constraints, respectively. Table 1 lists the simulation results showing the effect due to quantization errors with the maximum value $\eta=0.2$ for the case of unit gain constraint.

\subsection{Examples of eigenspace partially adaptive beamformers}

Similar to the above example, an incoherent interferer is incident from $20^{\circ}$ off broadside with INR $=10 \mathrm{~dB}$. Fig. 4(a) shows the WDF in $\mathrm{dB}$ versus the error variance $\sigma_{\mathrm{w}}^{2}$ for the case of the beamformer with unit gain constraint, while Fig. 4(b) shows the simulation results for the case of the beamformer with unit gain plus 1st derivative constraint. We used ideal signal covariance matrix $R_{X}$ for the simulations. The curves of $M=9$ and $M=8$ correspond to the GSC based fully adaptive beamformers with unit gain and unit gain plus 1st derivative constraints, respectively. The simulation results showing the effect of quantization errors are listed in Table 2 for the case of unit gain constraint and $\eta=0.2$.

\subsection{Examples of beam space partially adaptive beamformers}

In this case, the incoherent interferer is incident from $20^{\circ}$ off broadside with INR $=10 \mathrm{~dB}$. Fig. 5 shows the WDF in $\mathrm{dB}$ versus the error variance $\sigma_{\mathrm{w}}^{2}$ for the case of the beamformer with unit gain constraint. Again, ideal signal covariance matrix $R_{X}$ was used. The curve of $M=9$ corresponds to the GSC based fully adaptive beamformer with unit gain constraint. Table 3 lists the simulation results 

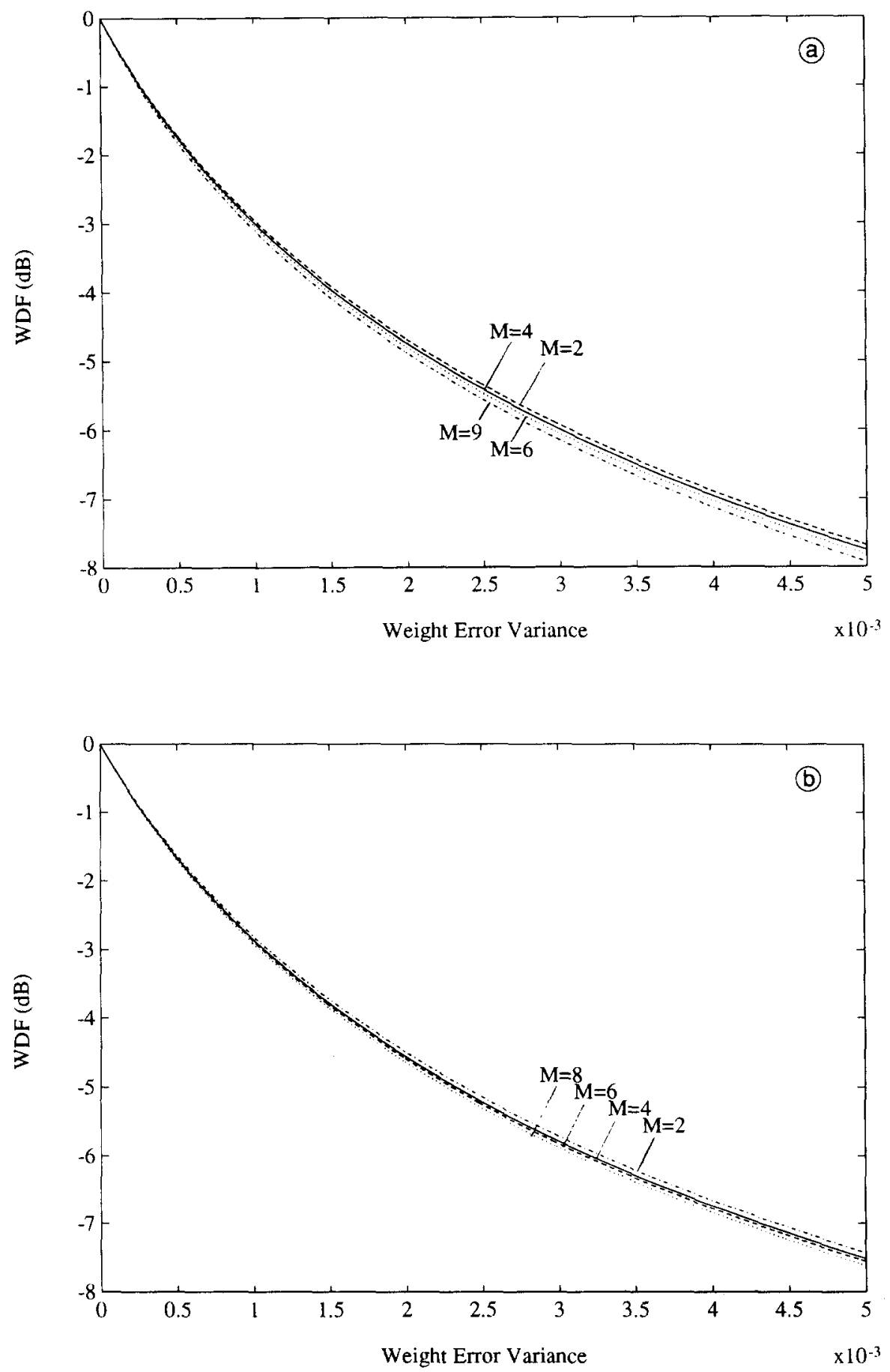

Fig. 4. The WDF versus the weight error variance $\sigma_{\mathrm{w}}^{2}$ for the eigenspace beamformer. (a) Unit gain constraint. (b) Unit gain plus 1st derivative constraint. 
Table 2

Effect of quantization errors for the case of eigenspace partially adaptive beamformers

\begin{tabular}{|c|c|c|c|c|}
\hline \multirow[b]{2}{*}{$\begin{array}{l}\text { Number of } \\
\text { bits } K\end{array}$} & \multicolumn{2}{|c|}{ Full adaptivity $(M=9)$} & \multicolumn{2}{|c|}{ Partial adaptivity $(M=6)$} \\
\hline & $\begin{array}{l}\text { Simulation result } \\
\text { SINR }=9.55 \mathrm{~dB} \\
\text { WDF (dB) }\end{array}$ & $\begin{array}{l}\text { Theoretical result } \\
\text { SINR }=9.90 \mathrm{~dB} \\
\text { WDF }(\mathrm{dB})\end{array}$ & $\begin{array}{l}\text { Simulation result } \\
\text { SINR }=9.67 \mathrm{~dB} \\
\text { WDF }(\mathrm{dB})\end{array}$ & $\begin{array}{l}\text { Theoretical result } \\
\text { SINR }=9.90 \mathrm{~dB} \\
\text { WDF }(\mathrm{dB})\end{array}$ \\
\hline 11 & 0 & 0 & -0.0001 & 0 \\
\hline 9 & -0.0001 & -0.0005 & -0.0005 & -0.0004 \\
\hline 7 & -0.0104 & -0.0074 & -0.0121 & -0.0071 \\
\hline 5 & -0.2083 & -0.1162 & -0.1931 & -0.1130 \\
\hline 3 & -2.6500 & -1.5651 & -1.2006 & -1.5279 \\
\hline
\end{tabular}

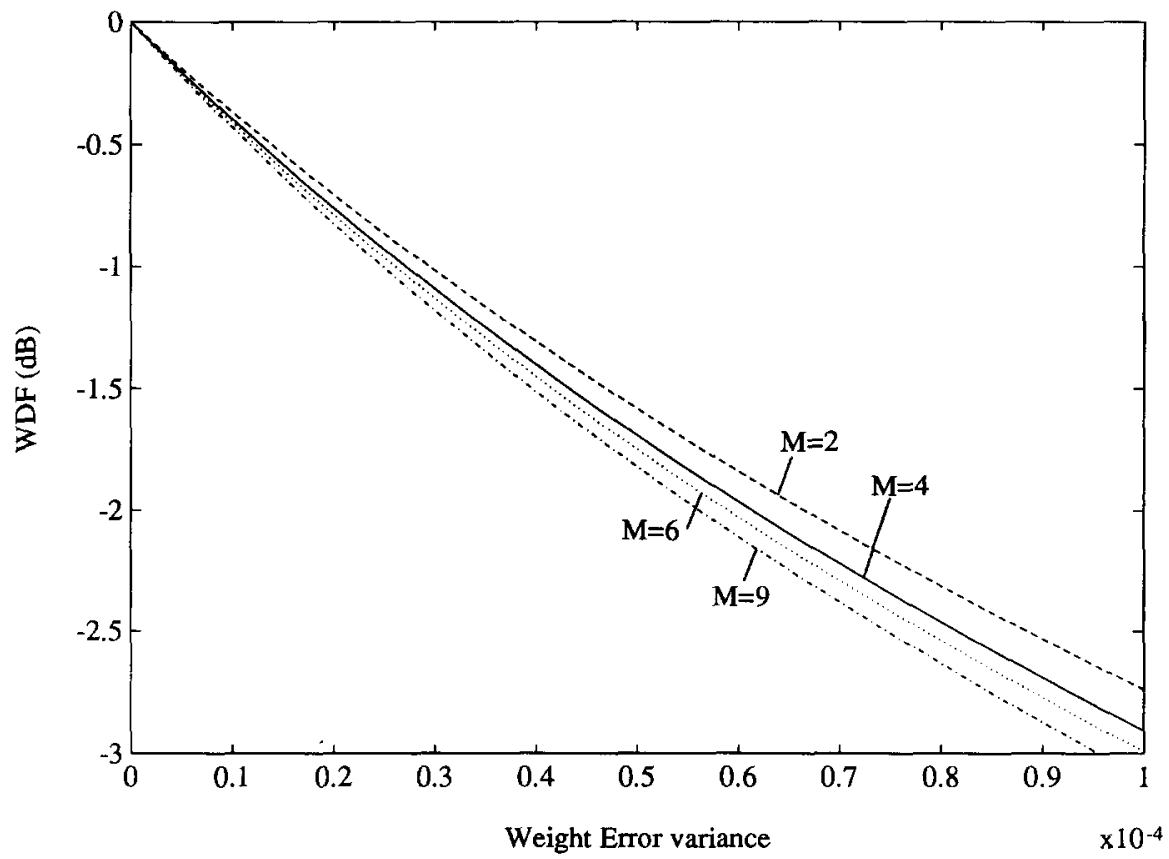

Fig. 5. The WDF versus the weight error variance $\sigma_{w}^{2}$ for the beam space beamformer with unit gain constraint.

showing the effect due to quantization errors with the maximum value $\eta=0.2$ for this case.

From these simulation results, it is confirmed that the array sensitivity to random weight errors increases as the number of the signal channels used for weight adaptation increases for each case of the three different beamformers. Using unit gain plus first derivative constraint causes severer performance degradation than using only unit gain constraint in the case of GSC based element space partially adaptive beamformers. Considering quantization errors, we note that the simulation results are very close to the theoretical results for the GSC based element space partially adaptive beamformers. However, the difference between the simulation result and the theoretical result increases significantly as the number of bits decreases for the GSC based eigenspace and beam space 
Table 3

Effect of quantization errors for the case of beam space partially adaptive beamformers

\begin{tabular}{|c|c|c|c|c|}
\hline \multirow[b]{2}{*}{$\begin{array}{l}\text { Number of } \\
\text { bits } K\end{array}$} & \multicolumn{2}{|c|}{ Full adaptivity $(M=9)$} & \multicolumn{2}{|c|}{ Partial adaptivity $(M=6)$} \\
\hline & $\begin{array}{l}\text { Simulation result } \\
\text { SINR }=9.55 \mathrm{~dB} \\
\text { WDF (dB) }\end{array}$ & $\begin{array}{l}\text { Theoretical result } \\
\text { SINR }=9.90 \mathrm{~dB} \\
\text { WDF }(\mathrm{dB})\end{array}$ & $\begin{array}{l}\text { Simulation result } \\
\text { SINR }=9.67 \mathrm{~dB} \\
\text { WDF }(\mathrm{dB})\end{array}$ & $\begin{array}{l}\text { Theoretical result } \\
\text { SINR }=9.89 \mathrm{~dB} \\
\text { WDF }(\mathrm{dB})\end{array}$ \\
\hline 11 & -0.0001 & -0.0003 & -0.0001 & -0.0003 \\
\hline 9 & -0.0043 & -0.0046 & -0.0038 & -0.0044 \\
\hline 7 & -0.0691 & -0.0730 & -0.0687 & -0.0695 \\
\hline 5 & -0.8744 & -1.0420 & -0.8469 & -0.9977 \\
\hline 3 & -4.8601 & -7.2743 & -4.9643 & -7.1030 \\
\hline
\end{tabular}

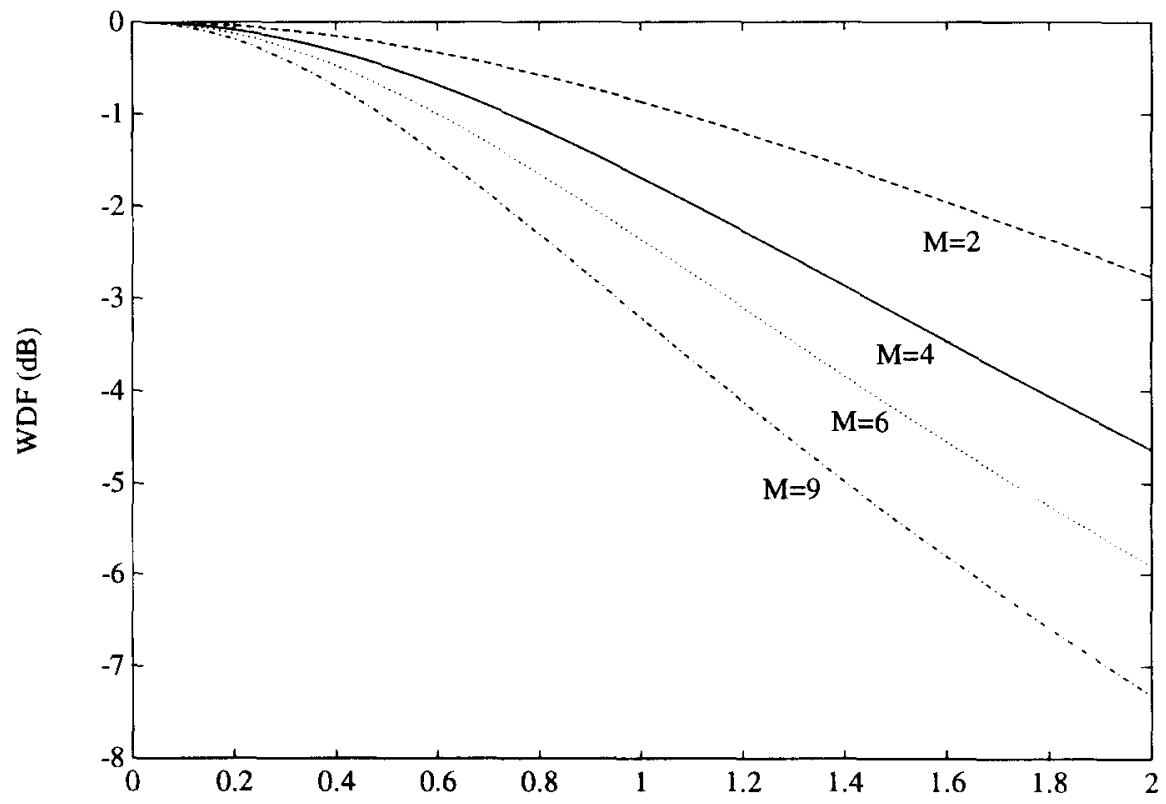

Fig. 6. The WDF versus the scaling factor $\delta$ of $B$ for the element space beamformer with unit gain constraint.

partially adaptive beamformers. This shows that the assumption of independently uniform distribution for modelling the random weight errors due to the quantization errors becomes rough as the number of bits decreases.

Next, we present experiments for illustrating the effect of the scale associated with the signal blocking matrix $B$ on the performance sensitivity of adaptive beamformers. Fig. 6 plots the WDF in dB versus the scaling factor $\delta$ of $B$, i.e., $B$ is replaced by $\delta B$ for the element space adaptive beamformers with unit gain constraint when the error variance $\sigma_{\mathrm{w}}^{2}$ is equal to 0.001. Experimental results showing the effect of quantization error versus $\delta$ for the element space partially adaptive beamformer with unit gain constraint and $M=4$ are listed in Table 4. We note that small $\delta$ alleviates the degradation due to quantization errors.

To examine how the WDF changes as the interference direction varies. We performed the 
Table 4

Effect of quantization errors for the element space partially adaptive beamformer with $M=4$

\begin{tabular}{llllll}
\hline & $\delta=0.5$ & & $\delta=1.0$ & \\
\cline { 2 - 3 } $\begin{array}{l}\text { Number of } \\
\text { bits } K\end{array}$ & $\begin{array}{l}\text { Simulation result } \\
\text { SINR }=9.65 \mathrm{~dB} \\
\text { WDF (dB) }\end{array}$ & $\begin{array}{l}\text { Theoretical result } \\
\text { SINR }=9.80 \mathrm{~dB} \\
\text { WDF }(\mathrm{dB})\end{array}$ & & $\begin{array}{l}\text { Simulation result } \\
\text { SINR }=9.66 \mathrm{~dB}\end{array}$ & $\begin{array}{l}\text { Theoretical result } \\
\text { SINR }=9.80 \mathrm{~dB} \\
\text { WDF (dB) }\end{array}$ \\
\hline 11 & 0 & 0 & -0.0001 & WDF (dB) \\
9 & -0.0002 & -0.0001 & -0.0001 & -0.0002 \\
7 & -0.0009 & -0.0008 & -0.0035 & -0.0034 \\
5 & -0.0100 & -0.0134 & -0.0539 & -0.0535 \\
3 & -0.2058 & -0.2103 & & -0.7068 & -0.7862 \\
\hline
\end{tabular}
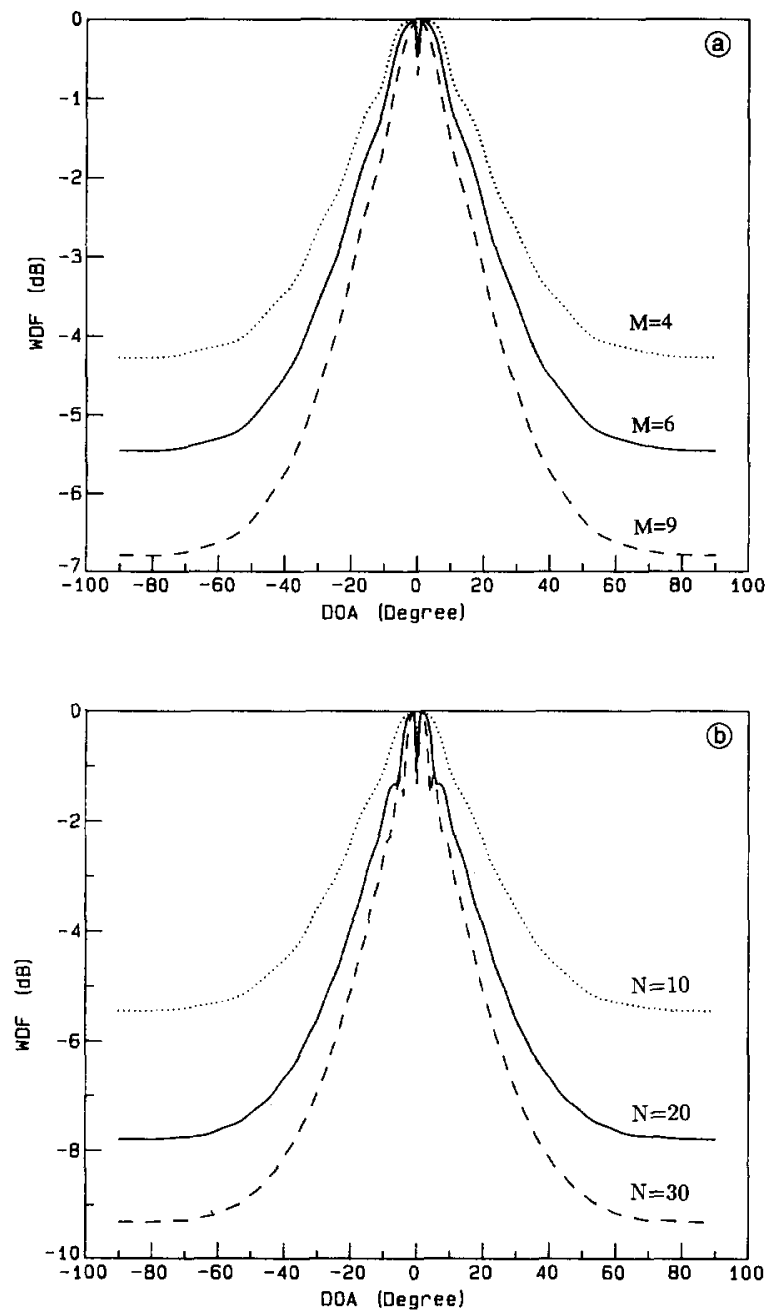

Fig. 7. The WDF versus the interference direction. (a) The number of array size $N=10$. (b) The number of adaptive size $M=6$. simulations of the element space partially adaptive beamformers with $\sigma_{\mathrm{w}}^{2}$ fixed at 0.001 . Fig. 7(a) plots the WDF versus the interference direction for different adaptive size $M$, while Fig. 7(b) plots the WDF versus the interference direction for different array size $N$. These figures show that the smaller the interferer's angle off broadside is, the larger the WDF is. This is because the interference impinging the array beamformer in the region near the broadside makes $\alpha_{i}$ small and hence increases the WDF of (32).

Finally, we present another simulation of the element space partially adaptive beamforming with two incoherent interferers impinging from $20^{\circ}$ and $30^{\circ}$ off broadside. The INRs are 20 and $30 \mathrm{~dB}$, respectively. Fig. 8 shows the resulting beampatterns with unit gain constraint and $M=6$ for the case without quantization and 7-bit (i.e., $K=7$ ) case. 1000 snapshots were used to compute the required signal covariance matrix. The null depths are about -63 and $-92 \mathrm{~dB}$ at the interference directions $20^{\circ}$ and $30^{\circ}$, respectively, for the case without quantization, while they are about -52 and $-47 \mathrm{~dB}$, respectively, for the 7-bit case.

\section{Conclusions}

In this paper, we have investigated the effect of random weight errors on the performance of adaptive array beamformers based on the GSC structure. First, analytical formulas of the array output SINR are derived for the element space, 


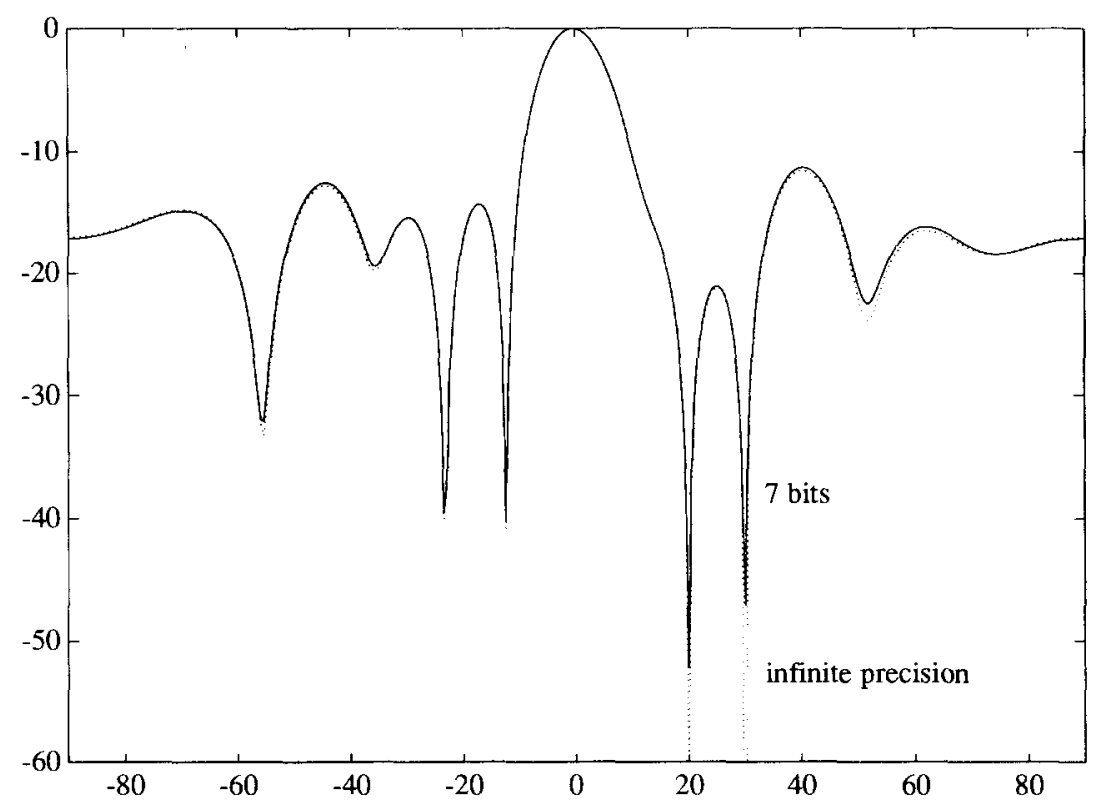

Fig. 8. The beampatterns of the element space partially adaptive beamformer with unit gain constraint and $M=6$. Dot line: the case without quantization; solid line: the 7-bit case.

eigenspace, and beam space beamformers with partial adaptivity, respectively. From these analytical formulas, we then define a weight degrading factor (WDF) for evaluating the effect of random weight errors on the performance of adaptive array beamformers. It has been shown theoretically that the sensitivity of adaptive beamformers to random weight errors increases as the number of adaptive weights used increases. Moreover, we also find from the theoretical analyses that the signal blocking matrix used in the GSC structure also affects the array sensitivity when random weight errors exist. The sensitivity can be reduced by designing a signal blocking matrix with smaller entries. Computer simulation examples including quantization errors for confirming the theoretical analyses are provided. We also observe from the simulations that using unit gain plus first derivative constraint causes severer performance degradation than using only unit gain constraint for GSC based element space partially adaptive beamformers.

\section{References}

[1] E. Brookner and J.M. Howell, "Adaptive-adaptive array processing", Proc. IEEE, Vol. 74, April 1986, pp. 602-604.

[2] D.J. Chaprnan, "Partial adaptivity for the large array", IEEE Trans. Antennas and Propagation, Vol. AP-24, September 1976, pp. 685-696.

[3] R.T. Compton, Jr., Adaptive Antennas, Concepts and Performance, Prentice-Hall, Englewood Cliffs, NJ, 1989.

[4] L.C. Godara, "Error analysis of the optimal antenna array processors", IEEE Trans. Aerospace Electron. Systems, Vol. AES-22, July 1986, pp. 395-408.

[5] L.J. Griffiths, "A new approach to partially adaptive arrays", Proc. Internat. Conf. Acoust. Speech Signal Process., Dallas, TX, USA, April 1987, pp. 1999-2002.

[6] L.J. Griffiths and C.W. Jim, "An alternative approach to linearly constrained adaptive beamforming", IEEE Trans. Antenras and Propagation, Vol. AP-30, January 1982, pp. $27-34$.

[7] A.M. Haimovich and Y. Bar-Ness, "An eigenanalysis interference canceller", IEEE Trans. Signal Process., Vol. 39, January 1991, pp. 76-84.

[8] T.T. Ma and L.J. Griffiths, "A solution space approach to achieving partially adaptive arrays", Proc. Internat. Conf. Acoust. Speech Signal Process., New York, USA, April 1988 , pp. $2869-2872$. 
[9] D.R. Morgan, "Partially adaptive array techniques", IEEE Trans. Antennas and Propagation, Vol. AP-26, November 1978, pp. 823-833.

[10] R. Nitzberg, "Effect of errors in adaptive weights", IEEE Trans. Aerospace Electron. Systems, Vol. AES-12, May 1976, pp. 369-373.

[11] N.L. Owsley, in: S. Haykin, eds., Array Signal Processing, Prentice-Hall, Englewood Cliffs, NJ, 1985.

[12] K. Takao and K. Uchida, "Beamspace partially adaptive antenna", IEE Proc. Part H, Microwaves, Antennas and Propagation, Vol. 136, December 1989, pp. 439-444.

[13] B.D. Van Veen, "Partially adaptive beamformer design via output power minimization", IEEE Trans. Acoust. Speech
Signal Process., Vol. ASSP-35, November 1987, pp. 1524-1532.

[14] B.D. Van Veen, "Eigenstructure based partially adaptive array design", IEEE Trans. Antennas and Propagation, Vol. AP-36, March 1988, pp. 357-362.

[15] B.D. Van Veen, "Improved power minimization based partially adaptive beamformer design", Proc. Internat. Conf. Acoust. Speech Signal Process., New York, USA, April 1988, pp. 2973-2976.

[16] B.D. Van Veen, "An analysis of several partially adaptive beamformer designs", IEEE Trans. Acoust. Speech Signal Process., Vol. ASSP-37, February 1989, pp. 192-203. 